\title{
ABUNDANCES IN A HOT HORIZONTAL-BRANCH STAR IN THE OLD OPEN CLUSTER NGC/6791
}

\author{
R.C. PETERSON \\ Astrophysical Advances, Palo Alto, CA \\ and UCO/Lick Observatories, Santa Cruz, CA \\ AND \\ E.M. GREEN \\ Steward Observatory, University of Arizona, Tucson, AZ
}

\begin{abstract}
.
The open cluster NGC 6791 is more metal-rich than any other open cluster, yet several hot blue horizontal branch (BHB) stars are probable members. We have performed an abundance analysis of the coolest such star, 2-17, which has a radial velocity and a proper motion which support cluster membership. We find parameters typical of a metal-rich BHB star, with rotational velocity $=16 \pm 1 \mathrm{~km} / \mathrm{s}$, an effective temperature $T_{\text {eff }}=$ $7250 \pm 150 \mathrm{~K}, \log$ gravity $=3.5 \pm 0.5 \mathrm{dex}$, and an iron abundance more than twice solar, $[\mathrm{Fe} / \mathrm{H}]=+0.4 \pm 0.1$ dex. The light even- $\mathrm{Z}$ elements $\mathrm{Mg}$, $\mathrm{Ca}$, and $\mathrm{Si}$ are further enhanced, ruling out $\mathrm{A}$-star peculiarities. Its analysis thus establishes the cluster metallicity accurately, free from serious blending which is problematical in metal-rich giants. Abundances of $\mathrm{C}, \mathrm{N}$, and $\mathrm{O}$ suggest little mixing. Visual comparison of the spectra of red HB stars in the cluster with that of the field star $\mu$ Leo indicates that the spectra are indistinguishable except for stronger $\mathrm{CN}$ in the former.

The high iron abundance, the light-element overabundances, the spectral similarity to strong-lined field giants, and the extensive presence of hot stars all highlight the fact that this old open cluster provides an excellent template for the study of metal-rich extragalactic systems. Properties of cluster members should establish not only accurate abundances for comparison with scenarios of nucelosynthesis and star formation, but also provide a testbed for ideas of the production of hot stars in a metal-rich environment, and so for the generation of the ultraviolet upturn in elliptical galaxies.
\end{abstract}




\section{Introduction}

The open cluster NGC 6791 is now considered both the oldest and the most metal-rich known. Its age is $8-10$ Gyrs, twice as old as the canonical solar-metallicity cluster M67 (Garnavich et al. 1994; Demarque, Green, \& Guenther 1992; Tripicco et al. 1995). That its metallicity is significantly above solar is suggested from moderate-resolution spectroscopy and from a mismatch of its color-magnitude diagram (CMD) with solar-metallicity isochrones. Tripicco et al. (1995) find $[\mathrm{Fe} / \mathrm{H}]=+0.27$ to +0.44 .

The cluster population is rich. In addition to about a dozen red giants and two dozen red horizontal-branch stars, the cluster has several very hot HB stars (Kaluzny \& Udalski 1992). Liebert et al. (1994) have shown that the extremely blue stars are mostly $\mathrm{sdB} / \mathrm{sdO}$ stars and at least 3 or 4 are likely cluster members, the first ever discovered in an open cluster. These may provide the key to the puzzling upturn in ultraviolet flux below $1500 \AA$ seen in many high-metallicity systems (Burstein et al. 1988; Ferguson et al. 1991; Rich, Minniti, \& Liebert 1993).

Cooler stars are less certain as members because of heavy field contamination. One is an almost certain member, however, with both a radial velocity and a preliminary proper motion (Cudworth 1994) which is consistent with membership. This star, $2-17$, has $\mathrm{B}-\mathrm{V}=0.37$ and $\mathrm{V}=15.0$, placing it at the extreme red end of the blue horizontal branch (BHB). Its analysis was undertaken to confirm its status as an evolved metal-rich BHB star and to establish abundances for the cluster as a whole from a spectrum in which line strengths and line blending are quite similar to that of the Sun, greatly reducing systematic errors.

\section{Abundance Analysis}

Echelle spectra of $30,000 \mathrm{FWHM}$ resolution were obtained on the $4 \mathrm{~m}$ telescope of the National Optical Astronomical Observatories at Kitt Peak near Tucson, AZ, with a TEK 2048x2048 CCD detector. Multiple exposures totaling several hours produced a spectrum with $\mathrm{S} / \mathrm{N}=30$ per pixel near $6000 \AA$ and covering $4500-7800 \AA$. The spectrum was reduced following standard procedures using the package IRAF. After cosmic-ray removal, dark and bias were removed by subtracting coadded sequences of dark and bias calibration frames. Flatfielding was accomplished by pixel-by-pixel division of the coadded spectrum by the coadded sequence of quartz lamp exposures. The wavelength solution was found by a two-dimensional fit to Th-Ar emission features and shifted by $52 \mathrm{~km} / \mathrm{s}$ to the stellar rest frame.

The abundance analysis proceeded in three stages: spectral synthesis to identify unblended FeI and FeII lines; an equivalent width analysis of these lines to establish stellar temperature $T_{\text {eff }}$, gravity $\log g$, and $[\mathrm{Fe} / \mathrm{H}]$, 
the logarithmic iron abundance with respect to the Sun; and spectral synthesis of the entire $5000-6500 \AA$ and $7500-7800 \AA$ region plus isolated additional sections to derive abundances for additional elements. Models interpolated in the grid of Kurucz (1993) were used with species number densities calculated explicitly for the elemental distribution involved. We employed the Kurucz programs SYNTHE and WIDTH.

For the spectral synthesis, atomic line parameters in the $5000-7500 \AA$ region were taken directly from the fit to the solar intensity spectrum by Peterson, Dalle Ore, \& Kurucz (1993), hereafter PDOK. To verify parameters for lines outside this wavelength region, calculations were also made for the solar intensity spectrum observed by Brault and reduced by Kurucz (see Kurucz 1991).

For the equivalent width analysis, we employed a set of $15 \mathrm{FeI}$ lines and 4 FeII lines in the $6000-6500 \AA$ region which were unblended and were included in the analysis of metal-poor giants by Kraft et al. (1995). Their equivalent widths in $2-17$ ranged from 15 to $112 \mathrm{~m} \AA$ for $\mathrm{FeI}$ and from 60 to $103 \mathrm{~m} \AA$ for FelI. We ran these lines twice through each model, using first the transition probabilities from Kraft et al. and then those of PDOK, to derive stellar $v_{\mathrm{t}}$ and $T_{\text {eff }}$ from the dependence of the deduced iron abundance on equivalent width and lower excitation potential. The results were as follows: for the Kraft et al. transition probabilities, $T_{\text {eff }}=$ $7330 \mathrm{~K}, v_{\mathrm{t}}=2.5 \mathrm{~km} / \mathrm{s}, \log (\mathrm{Fe} / \mathrm{H})=-3.96$, and $\operatorname{logg}=4.0$; for the PDOK transition probabilities, $T_{\text {eff }}=7250, v_{\mathrm{t}}=2.8 \mathrm{~km} / \mathrm{s}, \log (\mathrm{Fe} / \mathrm{H})=-3.98$, and $\log g=3.5$. The uncertainty in the mean in both cases is about $80 \mathrm{~K}$ for $T_{\text {eff }}$, $0.5 \mathrm{dex}$ for $\log g, 0.06$ in $\log (\mathrm{Fe} / \mathrm{H})$, and $0.3 \mathrm{~km} / \mathrm{s}$ for $v_{\mathrm{t}}$, with the $T_{\text {eff }}$ and $v_{t}$ uncertainties dictating that $\log (\mathrm{Fe} / \mathrm{H})$. The one-sigma deviation of a single line about the mean for FeI and FeII respectively for FeI and FeII respectively was 0.16 and 0.07 for the Kraft et al. values and 0.13 and 0.06 for those from PDOK, which for FeI probably reflect the uncertainties in the transition probabilities themselves. Their systematic uncertainties are difficult to estimate, and seem to dictate the overall uncertainty in $[\mathrm{Fe} / \mathrm{H}]$. Kraft et al. adopt $\log (\mathrm{Fe} / \mathrm{H})=-4.48$ for the Sun, while PDOK adopt the earlier Grevesse \& Anders (1989) value of -4.33 ; thus the relative iron abundance is $[\mathrm{Fe} / \mathrm{H}]=+0.51$ from the Kraft analysis, while $[\mathrm{Fe} / \mathrm{H}]=+0.35$ results from the $\mathrm{PDOK}$ analysis.

Subsequent spectral synthesis for the star was done using a model with $T_{\text {eff }}=7250, \log g=3.0, v_{\mathrm{t}}=2.7 \mathrm{~km} / \mathrm{s}$, and $[\mathrm{Fe} / \mathrm{H}]=+0.3$ and PDOK line parameters. This yielded abundances good to 0.1 dex for several additional species. $\mathrm{Mg}$ and $\mathrm{Si}$ are enhanced by 0.25 dex with respect to iron and $\mathrm{Ca}$ by $0.1 \mathrm{dex}$, while $\mathrm{Na}$ is enhanced by $0.4 \mathrm{dex}$. The very weak OI triplet at $6156 \AA$ indicates $[\mathrm{O} / \mathrm{Fe}]=0.0$, while the the strong $7774 \AA$ OI triplet is matched by $[\mathrm{O} / \mathrm{Fe}]=+0.2$; the latter is often anomalously strong in stars 
of such temperature. Three $\mathrm{CI}$ lines suggest $[\mathrm{C} / \mathrm{Fe}]=0.0$, while two $\mathrm{NI}$ features indicate $[\mathrm{N} / \mathrm{Fe}]=+0.5$.

The abundance analysis demonstrates that 2-17 has the properties expected of a metal-rich blue HB star. Its radial velocity agrees to wtihin 2 $\mathrm{km} / \mathrm{s}$ with that of the cluster mean, and was constant to $1 \mathrm{~km} / \mathrm{s}$ during the two nights of echelle observations. Its rotational velocity is $16 \mathrm{~km} / \mathrm{s}$, typical of blue HB stars in globular clusters (Peterson, Rood, \& Crocker 1995). Its overall iron abundance is high. Its relative abundance distribution shows no sign of chemical pecularities typical of Population I A stars; for example, calcium is underabundant in such stars.

The relative abundance distribution suggests that little if any mixing has occurred which might have altered $\mathrm{C}, \mathrm{N}$, or $\mathrm{O}$ abundances, since $[\mathrm{C} / \mathrm{Fe}]$ $=0$. We cannot rule out the possibility of an initially high $[\mathrm{C} / \mathrm{Fe}]$ value and subsequent reduction of $\mathrm{C}$ and $\mathrm{O}$ with enhanced $\mathrm{N}$ the result. However, a simpler interpretation is that the star reflects the abundance distribution of products of a type II supernova of solar metallicity or higher, in that it shows to a modest extent the enhancement of light even- $Z$ species which is ubiquitous in halo stars and attributed to this process (Wheeler, Sneden, \& Truran 1989). The $\mathrm{Na}$ and $\mathrm{N}$ enrichment could be the natural consequence of the high metallicity of the supernova.

\section{Spectral Comparison of Red Giants}

A subsequent run with the echelle spectrograph of Lick Observatory at the $3 \mathrm{~m}$ telescope on Mt. Hamilton during four nights in July and August 1997 yielded spectra of 35,000 resolution and S/N typically 20 per pixel, for nearly thirty stars whose colors suggested they are red HB members of NGC 6791. Radial velocities confirmęd this in almost all cases. Seven such spectra were coadded pixel-by-pixel to produce a preliminary composite spectrum typical of such stars.

The resulting spectrum was overlaid on a high $\mathrm{S} / \mathrm{N}$ spectrum of similar resolution previously obtained for the field star $\mu$ Leo, the prototypical super-metal-rich star. The result was surprising: details of spectral absorption lines matched extremely well. The only discernible difference appeared where $\mathrm{CN}$ features dominated; these were somewhat stronger in the composite NGC 6791 spectrum than in $\mu$ Leo.

\section{Conclusions}

In all the above aspects, the cluster NGC 6791 seems to provide a template for the study of metal-rich extragalactic systems. The iron abundance of the cluster is well established by the blue HB star 2-17, and is high, $0.35-0.5$ dex. The abundance distribution of that star probably reflects that of the 
cluster to a large extent; it is most simply interpreted as the result of the products of Type II supernova explosions whose progenitors were of solar metallicity or higher. The cluster's RHB stars are at least as strong-lined as the field super-metal-rich prototype $\mu$ Leo. Sodium and magnesium are strong features in such stars, as they are in the integrated visible spectra of luminous external galaxies. Moreover, the cluster harbors extremely blue $\mathrm{sdB} / \mathrm{sdO}$ stars, which could be the cause of the upturn in ultraviolet flux seen in the most luminous ellipticals.

The cluster serves as a caution to a straightforward scenario of heavyelement production within the Milky Way. It is old, but extremely metalrich, defying an age-metallicity relation. It seems difficult to produce such a high heavy-element abundance in situ, especially if Type II supernovae were involved, since the products would escape the cluster's small potential well. However, if formed by collisions among enriched molecular clouds, or as a merger with an external metal-rich object, it remains difficult to see how the fragile binding energy could be maintained. Given its orbit not far from the disk of the galaxy, its dynamical stability is a general issue.

The cluster's individual members pose similar challenges to stellar evolution and stellar population studies. Production of both $\mathrm{sdO} / \mathrm{sdB}$ stars and a BHB of intermediate temperature is very difficult with single-star evolution; perhaps binary mechanisms are involved, or the triggering of extreme mass loss by another mechanism. The colors of 2-17 point to the need for broader coverage in surveys of the Galactic disk designed to pick up blue stars. Because of its heavy line blanketing (due to its high metallicity), the visual colors are redder than normal for a stable HB star but rather typical of an RR Lyrae star; as such it might have been missed in many $\mathrm{BHB}$ surveys.

\section{References}

Burstein, D., et al. 1988, ApJ, 328, 440

Cudworth, K. M. 1994, private communication

Demarque, P., Green, E. M., \& Guenther, D. B. 1992, AJ, 103, 151

Ferguson, H. C., et al. 1991, ApJ, 382, L69

Friel, E. D., \& Janes, K. A. 1991, A\&A, 267, 75

Garnavich, P. M., VandenBerg, D. A., Zurek, D., \& Hesser, J. E. 1994, AJ, 107, 1097

Grevesse, N., \& Anders, E. 1989, in Cosmic Abundances of Matter, AIP Conf. 183, 1

Kaluzny, J., \& Udalski, A. 1992, A\&A, 42, 29

Kraft, R. P., Sneden, C., Langer, G. E., Shetrone, M. D., \& Bolte, M. 1995, AJ, 109,2586 
Kurucz, R. L. 1991, Rev. Mex. A. Ap.

Kurucz, R. L. 1993, CDROM series

Liebert, J., Saffer, R. A., \& Green, E. M. 1994, AJ, 107, 1408

Peterson, R. C., Dalle Ore, C. D. M., \& Kurucz, R. L. 1993, ApJ, 404, 333

Peterson, R. C., Rood, R. T., \& Crocker, D. A. 1995, 453, 214

Rich, R. M., Minniti, D., \& Liebert, J. 1993, ApJ, 406, 489

Tripicco, M. J., Bell, R. A., Dorman, B., \& Hufnagel, B. 1995, AJ, 109, 1697

Wheeler, J. C., Sneden, C., \& Truran, J. T. 1989, ARA\&A, 26 